\title{
PREVENÇÃO DAAIDS ENTRE ESTUDANTES UNIVERSITÁTRIOS: EXISTE INFLUÊNCIA DOS PARES?
}

\author{
AIDS PREVENTION AMONG COLLEGE STUDENTS: DO THEIR PEERS INFLUENCE THEM?
}

Antonio Carlos Gil1 \& Edmea Rita Temporini²

\begin{abstract}
'Doutor em Ciências Sociais da Fundação Escola de Sociologia e Política de São Paulo e em Saúde Pública-USP. ²Livre-Docente da Faculdade de Saúde Pública-USP. Assessora de Pesquisa. Disciplina de Oftalmologia - UNICAMP.

Correspondência: Profa. Dra. Edméa Rita Temporinl. Departamento de Prática de Saúde Pública. Faculdade de Saúde-USP. Avenida Doutor Arnaldo, 715. CEP 01246-904 São Paulo, São Paulo, Brasil.
\end{abstract}

GIL AC \& TEMPORINI ER. Prevenção da aids entre estudantes universitários: existe influência dos pares? Medicina, Ribeirão Preto, 33: 147-154 abr./ jun. 2000.

RESUMO: Modelo do estudo. Transversal analítico. Objetivo do estudo. Verificar percepções de estudantes da Grande São Paulo acerca de comportamentos de risco assumidos por seus pares, grau de risco dos colegas de contrair aids, sua competência para usar preservativo e situações em que aceitam a influência de seus pares na prevenção da aids. Metodologia. Foi elaborado um questionário auto-aplicável, após estudo exploratório. A amostra foi constituída por 1039 estudantes universitários, solteiros, de instituições particulares de ensino da Região da Grande São Paulo, obtida por critério de conveniência. Resultados. Em relação a comportamentos de risco assumidos por colegas, 56,2\% dos homens e 56,4\% das mulheres referem uso eventual de preservativo. Sexo com muitos parceiros foi admitido por $12,2 \%$ dos homens e $5,7 \%$ das mulheres. Quanto ao proprio risco de contrair aids, foi baixo o grau de percepção e menos acentuado entre as mulheres. Conclusões. Apesar de reconhecerem o uso de preservativo pelos pares a fim de evitar a aids, parece haver alguma incoerência nas percepções próprias acerca dos riscos de contrair aids, e nas dos colegas.

Unitermos: Síndrome de Imunodeficiência Adquirida. Prevenção \& Controle. Risco. Estudantes.

\section{1- INTRODUÇÃO}

Enquanto não se dispuser de uma vacina ou de tratamento que conduza à efetiva cura da Síndrome da Imunodeficiência Adquirida (aids), a adoção de medidas preventivas continuará sendo a resposta mais adequada ao combate dessa epidemia. A adoção de medidas preventivas eficazes, por sua vez, deve ser buscada individual e coletivamente, requerendo não apenas o conhecimento das formas de contágio pelo Vírus da Imunodeficiência Humana (HIV), mas, também, habilidade para efetivar essas medidas e atitudes favoráveis à sua adoção. Assim, por estar direta- mente relacionada a conhecimentos, habilidades e atitudes, a educação deve ser considerada como o mais efetivo instrumento de que se dispõe para a viabilização da prevenção da aids, o que, no entanto, representa novos desafios teóricos e práticos para a efetivação das intervenções educativas.

Ao contrário de muitas outras epidemias, a aids pode ser evitada, desde que se eliminem os comportamentos capazes de produzir a infecção pelo HIV, que são, principalmente, as relações sexuais e a troca de sangue por compartilhamento de seringas. Assim, à medida que se puder eliminar os comportamentos de risco, os casos de aids tenderão a diminuir. 
A abstinência de relações sexuais ou do uso de drogas injetáveis é vista como medida altamente eficaz para a prevenção da aids. Sua viabilização, todavia, é improvável. O que se pode propor mais realisticamente é a adoção de condutas sexuais preventivas como, por exemplo, usar preservativos e evitar manter relações sexuais com muitos parceiros. Tais propostas, no entanto, apresentam vários pontos críticos. A sugestão de sexo com um único parceiro poderá ser entendida por muitos segmentos sociais como extremamente moralista. A orientação para o uso de preservativos, por sua vez, poderá ser interpretada como promoção da promiscuidade.

Também há que se considerar que as práticas sexuais derivam de impulsos biológicos aos quais se torna difícil resistir. A atração sexual constitui um componente bastante valorizado na cultura juvenil. A atividade sexual pode ocorrer espontaneamente, quando o julgamento está ofuscado pelo consumo de álcool. Os preservativos podem ser rejeitados por serem antiestéticos, incômodos ou por dificultarem o prazer. A troca de seringas, por sua vez, tem sido entendida como elemento da subcultura dos consumidores de $\operatorname{drogas}^{(1)}$.

Embora se reconheça que a educação constitua a mais poderosa medida para a prevenção da aids, sua implementação mostra-se difícil, quando seu objetivo é mudar comportamentos de risco. Tem sido registrado o sucesso de programas objetivando a adoção de cintos de segurança, cuidados na condução de veículos, realização de exercícios físicos ou o abandono do fumo e do álcool, entre outros. A avaliação do sucesso de um programa relacionado à conduta sexual preventiva, entretanto, mostra-se muito mais complexa, pois não basta saber quantas pessoas participaram de cursos ou palestras ou como reagiram a elas. A efetiva avaliação requer que se saiba em que medida o programa foi capaz de promover mudanças na conduta sexual preventiva da população-alvo.

O conhecimento por si só não é um bom preditor de comportamentos preventivos ${ }^{(2)}$. A educação afetiva parece contribuir mais eficazmente para a mudança de comportamentos e atitudes ${ }^{(3)}$. Logo, um programa destinado a provocar mudanças comportamentais deve fundamentar-se em objetivos afetivos (direcionados a valores e motivações), apresentar um conteúdo programático ajustado a esses objetivos, utilizar estratégias e recursos de ensino reconhecidamente eficazes e também valer-se de técnicas apropriadas para aferir mudanças de atitudes. Vale, então, indagar até que ponto a aceitação e incorporação de práticas preventivas à contaminação HIV/aids dependeria da influência exercida por indivíduos que fazem parte do mesmo grupo social.

Os programas de prevenção da aids vêm incorporando, com freqüência cada vez maior, o princípio da educação pelos pares, mediante o qual os membros de um determinado grupo informam seus colegas acerca da transmissão do HIV e das medidas preventivas apropriadas ${ }^{(4 / 7)}$.

Coleman \& $\operatorname{Ford}^{(8)}$, a partir de revisão bibliográfica referente a programas de prevenção, implementados entre 1987 e 1995, nos Estados Unidos, destacaram a influência dos pares entre os principais fatores que contribuem para sua eficácia, enfatizando que a educação pelos pares, para todos os grupos e em todos os contextos culturais, constitui a mais efetiva estratégia de intervenção na luta contra a aids.

Cabe lembrar que as pessoas são mais suscetíveis de serem influenciadas sob condições imperfeitas de informação ${ }^{(9)}$. Considerando que as informações acerca da aids têm sido freqüentemente oferecidas de maneira contraditória, é de se admitir que as pessoas possam ser facilmente influenciadas, sobretudo por aquelas pessoas que integram os grupos primários de relacionamento. Além disso, a confiança na fonte de informação constitui importante precursor da influência. Diversos estudos sociológicos, por sua vez, demonstraram que a percepção e a aceitação do risco têm suas raízes em fatores sociais e culturais. Para Short ${ }^{(10)}$, por exemplo, a resposta ao risco é mediada, em especial, por influência social de amigos, família e colegas de trabalho.

A eficácia de qualquer programa fundamentado na educação pelos pares depende, no entanto, da percepção que os membros dos grupos têm em relação a seus pares, já que a estes caberia, em programas dessa natureza, proporcionar as informações sobre o contágio pelo HIV, bem como estimular atitudes favoráveis à adoção de condutas preventivas.

Nesse sentido, conceito derivado da Teoria da Aprendizagem Social de Bandura ${ }^{(11)}$, definido como "self efficacy", foi aplicado ao objeto deste estudo. Assim, pode ser definido como a confiança do indivíduo, proveniente de conhecimento e habilidade para a utilização correta do preservativo, visando à prevenção da aids. Essa definição pode ser entendida no emprego do termo "competência", opção deste trabalho. 
Existem vários modelos de educação em saúde, propondo a educação por pares e apresentando razões para tal. Contudo, existem limitações teóricas na maioria desses modelos e reduzida evidência empírica, relacionada à prática da promoção em saúde para apoiá-las. Desse modo, a educação realizada por colegas constitui ainda um campo de pesquisa, ao invés da aplicação da teoria à prática ${ }^{(12)}$.

Considerando esses aspectos, esta pesquisa teve por objetivo identificar percepções de estudantes da Grande São Paulo acerca de comportamentos de risco, assumidos por seus pares, grau de risco de colegas de contrair aids, sua competência para usar preservativo e situações em que aceitam a influência de seus pares na prevenção da aids.

\section{2- MÉTODO}

O delineamento adotado para o desenvolvimento desta pesquisa foi o transversal analítico, tendo em vista a intenção de obter conhecimento por meio de perguntas dirigidas ao grupo-alvo e de interpretar suas respostas conforme se apresentassem. A abordagem analítica foi possível mediante o cruzamento das variáveis selecionadas. A população foi constituída por estudantes universitários, matriculados em cursos das áreas de ciências exatas e humanas, em instituições particulares de ensino, situadas na região da Grande São Paulo. Excluiu-se da população o contingente de estudantes da área de ciências biológicas, admitindose possível exposição às informações sobre aids bem como os indivíduos casados.

A amostra foi selecionada pelo critério da intencionalidade. Procurou-se incluir, na amostra, estudantes de diferentes cursos, tanto em universidades quanto em estabelecimentos isolados de ensino superior. Procurou-se, ainda, garantir que a amostra apresentasse número equivalente de estudantes de ambos os sexos, tendo em vista a possível influência dessa variável no fenômeno em estudo.

O instrumento utilizado para coleta de dados foi um questionário auto-aplicado. Sua elaboração foi precedida de estudo exploratório na etapa de planejamento, buscando identificar variáveis relevantes, repertório de conhecimentos e vocabulário em relação ao tema ${ }^{(13)}$. Por meio desse recurso metodológico, construiu-se um instrumento estruturado (contendo perguntas e respostas) e adequado à realidade que se pretendia estudar. As informações que deram origem ao questionário definitivo foram obtidas mediante en- trevistas individuais e reuniões com grupos de estudantes que apresentavam características semelhantes às da população-alvo da pesquisa, sendo excluídos da amostra final. O questionário foi submetido a teste prévio, como prova de confiança e validade. $\mathrm{Na}$ aplicação do instrumento, anexou-se carta introdutória, apresentando os objetivos da pesquisa e instruções de preenchimento. Procurou-se, também, sensibilizar os estudantes a fornecerem as respostas, assegurando-se o anonimato e sigilo das respostas e o caráter voluntário da participação na pesquisa.

A coleta de dados foi realizada, em setembro e outubro de 1997, por pesquisadores de nível universitário. Responderam ao questionário os alunos presentes em sala de aula, não se observando recusas. Procedeu-se à revisão dos questionários, tendo sido excluídos os que apresentavam mais de cinco questões não respondidas, bem como os que foram respondidos por estudantes casados, considerando seu provável comportamento sexual mais estável e a aplicabilidade de certas questões. Os dados foram codificados, realizando-se seu processamento mediante a utilização do programa Epi-info 6.

Para a análise estatística das tabelas dos dados foi utilizado o teste Qui quadrado (x2 ). Como elemento adicional de análise, aplicou-se o valor escalar médio (VEM), que consiste na redução dos valores da distribuição a um único valor, tornando possível situar esse valor em uma escala e comparar entre si os valores de diversas escalas. Para tanto, atribuíram-se números às categorias das escalas ordinais, de acordo com a magnitude presumível do conteúdo implícito em cada categoria ${ }^{(14)}$.

O número de tabelas apresenta variações em função do número de respostas obtidas.

\section{3- RESULTADOS E DISCUSSÃO}

A amostra foi composta por 1039 estudantes, apresentando as seguintes características: 495 homens $(47,6 \%)$ e 544 mulheres $(52,4 \%)$; as idades estão compreendidas entre 17 e 38 anos, com média de 21,7 anos para o sexo masculino e 20,2 anos para o sexo feminino.

Os estudantes foram solicitados a informar acerca da percepção em relação a possíveis comportamentos de risco do colega com quem mais se relaciona: sexo sem camisinha, sexo com muitos parceiros, sexo com pessoas que têm muitos parceiros e uso de drogas injetáveis (Tabela I). 


\begin{tabular}{|c|c|c|c|c|c|}
\hline \multirow[b]{2}{*}{ Comportamentos dos pares } & & \multicolumn{4}{|c|}{ Sexo } \\
\hline & & \multicolumn{2}{|c|}{$\begin{array}{c}\text { Masculino } \\
\% \%^{*} \\
n=485\end{array}$} & \multicolumn{2}{|c|}{$\begin{array}{c}\text { Feminino } \\
\% \% \%^{*} \\
\mathrm{n}=538\end{array}$} \\
\hline \multicolumn{6}{|l|}{ Sexo com camisinha } \\
\hline (2) sempre & & 13,6 & 16,4 & 13,2 & 16,0 \\
\hline (1) às vezes sim, às vezes não & & 46,6 & 56,2 & 46,6 & 56,4 \\
\hline (0) nunca & & 22,7 & 27,4 & 22,9 & 27,6 \\
\hline - não sabe & & 17,1 & - & 17,3 & - \\
\hline & VEM $^{* *}$ & \multicolumn{2}{|c|}{0,89} & \multicolumn{2}{|c|}{0,88} \\
\hline & & \multicolumn{2}{|c|}{$\mathrm{n}=484$} & \multicolumn{2}{|c|}{$n=538$} \\
\hline \multicolumn{6}{|l|}{ Sexo com muitos parceiros } \\
\hline (2) sempre & & 9,9 & 12,2 & 3,0 & 5,7 \\
\hline (1) às vezes sim, às vezes não & & 38,0 & 46,6 & 16,5 & 20,4 \\
\hline (0) nunca & & 33,5 & 41,2 & 61,5 & 75,9 \\
\hline - não sabe & & 18,6 & - & 19,0 & \\
\hline VEM & & \multicolumn{2}{|c|}{$\begin{array}{l}0,71 \\
n=485\end{array}$} & \multicolumn{2}{|c|}{$\begin{array}{ll} & 0,31 \\
\mathrm{n} & =537\end{array}$} \\
\hline
\end{tabular}

Sexo com pessoas que têm muitos parceiros sexuais

\begin{tabular}{|c|c|c|c|c|}
\hline (2) sempre & 5,6 & 8,5 & 1,7 & 2,5 \\
\hline (1) às vezes sim, às vezes não & 27,6 & 41,9 & 14,7 & 21,6 \\
\hline (0) nunca & 32,6 & 49,6 & 51,6 & 75,9 \\
\hline - não sabe & 34,2 & - & 32,0 & - \\
\hline \multirow[t]{2}{*}{ VEM } & & 0,59 & & 0,26 \\
\hline & \multicolumn{2}{|c|}{$n=496$} & & $=542$ \\
\hline \multicolumn{5}{|l|}{ Uso de drogas injetáveis } \\
\hline (2) sempre & 0,2 & 0,3 & & \\
\hline (1) às vezes sim, às vezes não & 2,9 & 3,6 & 0,9 & 1,1 \\
\hline (0) nunca & 79,5 & 96,1 & 86,6 & 98,9 \\
\hline Não sabe & 17,4 & - & 12,5 & - \\
\hline VEM & \multicolumn{2}{|c|}{0,04} & \multicolumn{2}{|r|}{0,01} \\
\hline
\end{tabular}

* Exclui as respostas "Não sabe".

** Valor Escalar Médio - média ponderada dos valores atribuídos às categorias

A percepção acerca do comportamento de risco dos colegas varia em função do sexo. Nota-se, porém, que essa percepção é bastante influenciada pelos padrões reconhecidos como socialmente mais adequados. As moças reconhecem que suas colegas com freqüência mantêm relações sexuais sem camisinha $(84,0 \%)$, o que, embora constituindo comportamento de risco, não tem recebido, tradicionalmente, recriminação social. A percepção acerca da prática sexual exercida por colega com muitos parceiros é 
bem menos acentuada entre as moças do que entre os rapazes, já que 75,9\% delas admitem que suas colegas nunca adotam esse comportamento, contra apenas $41,2 \%$ dos rapazes. Também a percepção acerca da prática do sexo com pessoas que apresentam muitos parceiros é diversa entre moças $(75,9 \%)$ e rapazes $(49,6 \%)$. Já em relação ao uso de drogas injetáveis, que é recriminado, independentemente do sexo, as percepções são bastante semelhantes: 98,9\% das moças e $96,1 \%$ dos rapazes admitem que seus pares nunca adotam esse comportamento.

$\mathrm{Na}$ percepção desses estudantes, os colegas apresentam comportamento de risco em relação ao uso de camisinha, visto que, mais de metade afirma que eles ou elas praticam o sexo sem camisinha, sempre ou pelo menos ocasionalmente. As percepções acerca da prática do sexo com muitos parceiros ou com pessoas que têm muitos parceiros indicam comportamento de menor risco, embora os resultados indiquem, também, que os pares devam manter esses comportamentos não regularmente.

É interessante notar as diferenças de percepção, considerando a variável sexo. Em relação à prática do sexo com camisinha e o uso de drogas injetáveis, as percepções são muito semelhantes. No que se refere ao sexo com muitos parceiros e com pessoas que têm muitos parceiros sexuais, as moças tendem a admitir que suas colegas envolvem-se menos em situações de risco.

Essas diferenças de percepção explicam-se, possivelmente, por diferenças de conduta que a sociedade brasileira estabelece para os sexos, já que, tradicionalmente, confere-se maior liberdade ao homem do que à mulher no que se refere ao número de parceiros.

A percepção acerca do consumo de drogas injetáveis pelos colegas é bastante favorável, pois a grande maioria admite que seus colegas não fazem uso delas. Cabe considerar, entretanto, que, de todos os comportamentos considerados, este é o que apresenta maior conotação anti-social, já que envolve inclusive a possibilidade de sanções penais.

A percepção do risco a que os colegas estão submetidos em relação a aids distribui-se de acordo com a Tabela II.

Proporção relativamente reduzida de estudantes $(11,1 \%$ dos homens e $7,6 \%$ das moças) admite que seus colegas correm risco alto ou muito alto de contrair aids. O exame dos VEMs mostra que o risco percebido se situa entre os graus baixo e médio para os rapazes $(2,28)$, enquanto que, para as moças, o grau de risco percebido mostra-se menor $(1,89)$.

Os dados referentes a percepção de risco dos pares mostram como o risco de contrair aids parece ser pequeno, quando se trata de considerar o grupo social mais próximo. Isso indica não apenas que a aids é entendida como "doença dos outros", mas sobretudo como de "outros que estão distantes". Resultados semelhantes foram obtidos em pesquisas realizadas, no Brasil, com populações diversas, como estudantes do $2^{\text {o. }}$ grau $^{(15)}$ e universitários ${ }^{(14)}$.

A maioria dos estudantes de ambos os sexos acredita que seus colegas sabem usar camisinha e conseguem convencer o parceiro a usá-la, embora um número expressivo de estudantes tenha declarado desconhecimento (Tabela III).

De outro lado, os estudantes informam serem influenciados pelos colegas quanto à conduta preventiva em relação a aids (Tabela IV). O que mostra bastante coerência com as teorias que ressaltam a importância dos fatores de natureza social, tanto para o conhecimento quanto para o desenvolvimento de ações preventivas de saúde, como a Theory of reasoned action $^{(16)}$ e o modelo conhecido como Group based influence $e^{(17)}$. Ao adotarem comportamentos específico, esses estudantes são influenciados por grupos de referência. Fazendo o que os colegas fazem ou pensando como eles pensam, sentem-se aprovados socialmente, pelo menos em relação ao grupo social com que mais se identificam, que é o de seus colegas. Assim, se esses colegas adotam comportamentos de risco ou manifestam atitudes que os predispõem a adotá-los, tendem igualmente a adotá-los.

\begin{tabular}{|c|c|c|}
\hline $\begin{array}{l}\text { Percepção } \\
\text { de Risco }\end{array}$ & $\begin{array}{c}\text { Masculino } n=489 \\
\%\end{array}$ & $\begin{array}{c}\text { Feminino } n=541 \\
\%\end{array}$ \\
\hline (5) Muito alto & 3,1 & 2,2 \\
\hline (4) Alto & 8,0 & 5,4 \\
\hline (3) Médio & 33,5 & 31,1 \\
\hline (2) Baixo & 30,7 & 28,5 \\
\hline (1) Muito baixo & 19,0 & 25,0 \\
\hline (0) Nenhum & 5,7 & 7,8 \\
\hline VEM * & 2,28 & 1,89 \\
\hline $\begin{array}{l}\text { * Valor Escalar } \\
\text { atribuídos às cat }\end{array}$ & $\begin{array}{l}\text { lédio - média pon } \\
\text { gorias }\end{array}$ & erada dos valores \\
\hline
\end{tabular}




\begin{tabular}{|c|c|c|c|c|}
\hline \multirow{3}{*}{$\begin{array}{l}\text { Percepção acerca da competência dos pares: } \\
\text { Usar a camisinha com competência }\end{array}$} & \multicolumn{2}{|c|}{ Masculino $(n=490)$} & \multicolumn{2}{|c|}{ Feminino $(\mathrm{n}=538)$} \\
\hline & \multirow[t]{2}{*}{$\%$} & \multirow[t]{2}{*}{$\%$ * } & \multirow[t]{2}{*}{$\%$} & \multirow[t]{2}{*}{$\%$ * } \\
\hline & & & & \\
\hline $\operatorname{Sim}$ & 73,3 & 96,0 & 68,1 & 96,1 \\
\hline Não & 3,1 & 4,0 & 2,7 & 3,9 \\
\hline Não sabe & 23,6 & - & 29,2 & - \\
\hline \multicolumn{5}{|l|}{$\begin{array}{l}\text { Convencer o(a) parceiro(a) a manter relações } \\
\text { sexuais com camisinha }\end{array}$} \\
\hline Sim & 62,0 & 93,0 & 58,3 & 88,3 \\
\hline Não & 4,7 & 7,0 & 7,8 & 11,7 \\
\hline Não sabe & 33,3 & - & 33,9 & \\
\hline
\end{tabular}

* Exclui as respostas "Não sabe".

\begin{tabular}{|c|c|c|c|c|c|c|}
\hline \multirow[b]{2}{*}{ Situação de influência } & \multicolumn{3}{|c|}{ Masculino } & \multicolumn{3}{|c|}{ Feminino } \\
\hline & $\begin{array}{c}\text { Sempre } \\
\%\end{array}$ & $\begin{array}{c}\text { Às vezes } \\
\%\end{array}$ & $\begin{array}{c}\text { Nunca } \\
\%\end{array}$ & $\begin{array}{c}\text { Sempre } \\
\%\end{array}$ & $\begin{array}{c}\text { Às vezes } \\
\%\end{array}$ & $\begin{array}{c}\text { Nunca } \\
\%\end{array}$ \\
\hline \multirow[b]{2}{*}{ Ouve o que dizem } & \multicolumn{3}{|c|}{$\mathrm{n}=466$} & \multicolumn{3}{|c|}{$n=516$} \\
\hline & 60,0 & 37,3 & 2,7 & 61,7 & 35,6 & 2,7 \\
\hline \multirow{2}{*}{ Respeita as opiniões } & \multicolumn{3}{|c|}{$n=473$} & \multicolumn{3}{|c|}{$\mathrm{n}=518$} \\
\hline & 68,6 & 30,5 & 0,9 & 77,1 & 22,7 & 0,2 \\
\hline \multirow[b]{2}{*}{ Discute o assunto } & \multicolumn{3}{|c|}{$n=476$} & \multicolumn{3}{|c|}{$n=525$} \\
\hline & 69,0 & 28,0 & 0,2 & 65,7 & 32,4 & 1,9 \\
\hline \multirow[b]{2}{*}{ Faz o que sugerem } & \multicolumn{3}{|c|}{$n=460$} & \multicolumn{3}{|c|}{$n=508$} \\
\hline & 5,4 & 75,7 & 18,9 & 4,3 & 77,0 & 18,7 \\
\hline \multirow[b]{2}{*}{ Faz o que fazem } & \multicolumn{3}{|c|}{$n=460$} & \multicolumn{3}{|c|}{$n=508$} \\
\hline & 3,9 & 55,4 & 40,7 & 1,8 & 48,4 & 49,8 \\
\hline
\end{tabular}

No que se refere a ouvir o que dizem, respeitar as opiniões e discutir o assunto, a grande maioria respondeu "sempre". Já no que se refere a fazer o que sugerem e fazem, a maioria respondeu "às vezes". Note-se, ainda, que as moças declararam, com frequiência maior que a dos rapazes, respeitar a opinião dos colegas; no entanto, com freqüência menor, declararam fazer o que as demais fazem.

A grande quantidade de respostas afirmando nunca fazerem o que os colegas fazem pode ser entendida como reforçador da confiança que os estudantes têm em relação a sua conduta preventiva ou à necessidade de auto-afirmação característica desse grupo etário. À medida que admitem ser baixo o risco dos colegas contraírem aids e não fazerem o que fazem, seu risco pessoal tende a ser menor. Por outro lado, também parece razoável admitir que, ao afirmarem não fazer o que os colegas sugerem ou fazem, estão procurando afirmar sua independência, o que constitui importante valor na cultura juvenil.

Biglan et al. ${ }^{(18)}$, em pesquisa desenvolvida nos Estados Unidos, verificaram que os pares são capazes de influenciar o engajamento de adolescentes em muitas situações em que os comportamentos de risco 
se tornam mais prováveis. Metzler et al. ${ }^{(19)}$, também nos Estados Unidos, constataram que adolescentes cujos pares apresentavam envolvimento com problemas comportamentais apresentavam maior tendência a manifestar comportamento sexual de risco. Dolvanac $\&$ Zimmerman $^{(20)}$, após estudarem a relação entre comportamento de risco e fatores psicossociais, nos Estados Unidos, também constataram que os comportamentos de risco eram mais prováveis entre adolescentes que tinham pares envolvidos em condutas delinqüentes, como agressões, roubos, uso de álcool e drogas. Svenson \& Hanson ${ }^{(21)}$, com base nos resultados de pesquisa com universitários suecos, concluíram que a influência dos pares constitui importante componente a ser incluído nos programas de prevenção à aids.

Os resultados deste estudo indicam que os jovens admitem que seus colegam usam a camisinha com competência e são capazes de convencer seus parceiros a usá-la. No entanto, reconhecem que, mesmo assim, seus pares adotam comportamento de ris- co. A discussão deste aspecto pelos pares deve, portanto, ser encarada como importante estratégia a ser desenvolvida nos programas preventivos que têm o jovem como população alvo.

A influência dos pares em conduta de risco requer aprofundamento. As teorias a esse respeito são limitadas em seu escopo e apresentam fracas evidências empíricas $^{(12)}$. No entanto, os resultados deste estudo, associados a outros, tais como os citados, permitem sugerir que programas de prevenção da aids entre jovens incluam estratégias de preparação de lideranças para participarem das atividades de orientação de seus pares. Essa forma de aproximação poderá contribuir para a diminuição da exposição ao risco de contrair o HIV.

\section{AGRADECIMENTOS}

Agradecemos à Fundação para o Amparo à Pesquisa do Estado de São Paulo o auxílio financeiro concedido ao Projeto da pesquisa - Processo no 95/0041-9, que deu origem ao presente trabalho.

GIL AC \& TEMPORINI ED. Aids prevention among college students: do their peers influence them? Medicina, Ribeirão Preto, 33: 147-154, apr./june 2000.

ABSTRACT: Study design. Analytical survey. Study purpose. To check on the students' perceptions living in the Great São Paulo, State of São Paulo, Brazil, about risky behaviors adopted by their peers; the information about the proper risk for contracting aids, use of condom and the ability to insure their peers in using it and the circumstances in which they accepted being influenced by their peers concerning aids prevention. Methodology. After an exploratory study, a self-applied questionnaire was administered among students. The sample was obtained by a convenience criterion, constituted of 1,039 college students attending private schools in the Great São Paulo area. Results. As to peers risky behaviors $56,2 \%$ of men and $56,4 \%$ of women reported the inconsistent condom use; sexual intercourse with many partners was admitted by $12,2 \%$ of men and by $5,7 \%$ of women. As to their own risky behavior of contracting aids, the degree of perception was low, being less marked among women. Conclusions. Though acknowledging the condom use on the part of their peers as a means for avoiding aids for themselves and to their peers, it seems to exist some incoherence concerning their perception of risk contracting aids, their own and that of their peers.

UNITERMS: Acquired Immunodeficiency Syndrome. Prevention \& Control. Risk-Taking. Students.

\section{REFERÊNCIAS BIBLIOGRÁFICAS}

1 - FINEBERG HV. Education to prevent AIDS: prospects and obstacles. Science 239: 592-596, 1988.

2 - CATANIA JA; GIBSON DR; CHITWOOD DD \& COATES TJ. Methodological problems in AIDS behavioral research: influences on measurement error and participation bias in studies of sexual behavior. Psychol Bull 108: 339-362, 1990.

3 - Di CLEMENTE RJ; BOYER C \& MILLS S. Prevention of aids among adolescents: strategies for the development of riskreduction health education program. Health Educ Res 2: 287-291,1987. 
4 - JANS NK; ZIMMERMAN MA; WREN PA; ISRAEL BA; FREUDENBERG N \& CARTER R. Evaluation of 67 aids prevention projects: sucessfull approaches and barriers to programs effectiveness. Health Educ Q 23: 80-97, 1996.

5 - KELLY JA; St. LAWRENCE JS; DIAZ YE; STEVENSON LY; HAUTH AC; BRASFIELD TL; KALICHMAN SC; SMITH JE \& ANDREW ME. 1001. Risk behavior reduction following intervention with key opinion leaders of population: an experimental analysis. Am J Public Health 81: 168-171, 1991.

6 - REEDER GD; PRYOR JB \& HARSH L. Activity and similarity in safer Sex workshops led by peers educators. AIDS Educ Prev 9: 77-89, 1997. Suppl. A.

7 - RICKERT VI; JAY MS \& GOTTLIEB A. A comparison of three clinical-based aids education program on female adolescents' knowledge, attitudes and behavior. J Adolesc Health Care 44: 298-303, 1990.

8 - COLEMAN LM \& FORD NJ. An extrensive literature review of the evaluation of HIV prevention programmes. Health Educ Res 11: 327-338, 1996.

9 - PARSONS T. The social system. Free Press, New York, 1951.

10 - SHORT JF Jr. The social fabric at risk. Toward the social transformation of risk analysis. Am Sociol Rev 6: 711-725, 1984.

11 - BANDURA A. Self-efficacy: toward an unifying theory of behavioral change. Psychol Rev 84: 191-205, 1977.

12 - TURNER G \& SHEPHERD J. A method in search of a theory: peer education and health promotion. Health Educ Res 14: 235-247, 1999.

13 - PIOVESAN A \& TEMPORINI ER. Pesquisa exploratória: procedimento metodológico para o estudo de fatores humanos no campo da saúde pública. Rev Saúde Pública 29: 318325, 1995.
14 - TEMPORINI ER. Prevenção da aids: percepção e conduta sexual de estudantes universitários de São Paulo. Tese de Livre-Docência. Faculdade de Saúde Pública da USP, São Paulo, p. 29-32, 1995.

15 - CORDEIRO RGF \& TEMPORINI ER. Uso de preservativo para prevenção da aids: opinião e conduta de estudantes do $2^{\circ}$. grau. J Bras Doenças Sex Transm 9: 29-34, 1997.

16 - AJZEN I \& FISHBEIN M. Understanding attitudes and protecting social behavior. Prentice-Hall, Englewood Cliffs, 1980

17 - FISHER J \& FISHER W. Changing aids-risk behavior. Psychol Bull 11: 455-474, 1992

18 - BIGLAN A; METZLER CA; WIRT R; ARY D; NOELL J; OCHS L; FRENCH C \& HOOD D. Social and behavioral factors associated with high-risk sexual behavior among adolescents. J Behav Med 13: 245-262, 1990.

19 - METZLER CW; NOELL J; BIGLAN A; ARY D \& SMOLKOWSK $\mathrm{K}$. The social context for risk sexual behavior among adolescents. J Behav Med 17: 419-438, 1994.

20 - DOLVANAC RF \& ZIMMERMAN MA. Psychological factors and high-risk sexual behavior: race differences among urban adolescents. J Behav Med 21: 451-467, 1998.

21 - SVENSON GR \& HANSON BS. Are peer and social influences important components to include in HIV-STD prevention models? Results of a survey on young people at Lund University, Sweden. Eur J Public Health 6: 203-211, 1996.

Recebido para publicação em 25/01/2000

Aprovado para publicação em 03/05/2000 